\title{
On the GPS widelane and its decorrelating property
}

\author{
P. J. G. Teunissen \\ Delft Geodetic Computing Centre (LGR), Faculty of Geodesy, Delft University of Technology, Thijsseweg 11, 2629 JA Delft,
} The Netherlands Fax: + 3115278 3711; e-mail: lgr@geo.tudelft.nl

Received: 11 November 1996 / Accepted: 23 April 1997

\begin{abstract}
In this contribution we consider the popular widelaning technique from the viewpoint of ambiguity decorrelation. It enables us to cast the technique into the framework of the least-squares ambiguity decorrelation adjustment (LAMBDA) and to analyse its relative merits. In doing so, we will provide answers to the following three questions. Does the widelane decorrelate? Does it explicitly appear in the automated transformation step of the LAMBDA method? Can one do better than the widelane? It is shown that all three questions can be answered in the affirmative. This holds true for the ionosphere-fixed case, the ionosphere-float case, as well as for the ionosphere-weighted case.
\end{abstract}

Key words. GPS · Ambiguity decorrelation .

Widelane $\cdot$ Ionosphere

\section{Introduction}

Fast and high-precision GPS relative positioning often relies on one's ability to resolve quickly the integer values of the double-differenced (DD) carrier-phase ambiguities. This topic has therefore been a rich source of GPS research over the last decade or so, resulting in a variety of different methods and proposals for efficiently estimating the integer ambiguities, see e.g. Counselman and Gourevitch (1981), Hatch (1982, 1989), Remondi (1986), Blewitt (1989), Frei (1991), Wübbena (1989, 1991), Allison (1991), Cocard and Geiger (1992), Euler and Landau (1992), Goad (1992), Mervart et al. (1994).

When dual-frequency data are available, many of the existing techniques make good use of the popular widelaning technique. For the purpose of integer ambiguity estimation, the widelane phase observable, with its relatively long wavelength, relatively low noise behaviour and relatively small ionospheric delay, is considered a useful linear combination. The ambiguity of the wide- lane observable is the difference of the $L_{1}$ and $L_{2} \mathrm{DD}$ ambiguity. The least-squares ambiguity decorrelation adjustment (LAMBDA), introduced in Teunissen (1993), also makes use of integer linear combinations of the original DD ambiguities. The transformation step of this method is based on constructing integer linear combinations of the DD ambiguities, such that new ambiguities are obtained which are more precise and less correlated than the original DD ambiguities. Examples of its performance can be found in e.g. Teunissen (1994, 1995a), Teunissen and Tiberius (1994), Jonge and Tiberius (1996).

It is the goal of the present contribution to show how the by now classical, but still often used, widelaning technique fits into the framework of the LAMBDA method. For that purpose, our study will give answers to the following three questions: (1) Does the widelane decorrelate? (2) Does the widelane appear in the transformation step of the LAMBDA method? (3) Can one do better than the widelane?

In order to be able to answer these three questions, we first give a very brief review of the concepts underlying the ambiguity decorrelation; this is done in Sect. 2. In Sect. 3, we will answer the first question; there, we also present the ambiguity variance matrix for the case in which the ionospheric delays are assumed absent or known, for the case in which the ionospheric delays are assumed present but completely unknown, and for the case in which the ionospheric delays are treated as random variables. In Sect. 4, the second and third question are answered. The transformation that produces the widelane is compared to the sequence of steps that builds up the decorrelating ambiguity transformations. The section is concluded with a number of numerical examples.

\section{Integer ambiguity estimation in two dimensions}

For the purpose of the sections following, we will give a brief review of the least-squares ambiguity decorrelation adjustment. Attention will be restricted to the twodimensional case. 


\subsection{Integer least-squares}

The linear(ized) GPS model of observation equations on which the estimation of the integer ambiguities is based is generally of the form

$y=A a+B b+e$

where $y$ is a vector of 'observed minus computed' DD GPS observables, $a$ is the vector of unknown integer DD ambiguities, $b$ is a vector that includes all remaining unknown parameters and $e$ is the vector that takes care of the measurement noise and remaining unmodelled effects. The matrices $A$ and $B$ are the appropriate design matrices.

In order to solve for this system of equations, the least-squares principle is applied. Since the ambiguities are known to be integer, we are dealing with an integer least-squares problem rather than a standard leastsquares problem. The integer least-squares problem can be solved in three steps. First, an ordinary least-squares solution is computed. Hence, in this step the integer constraints on the ambiguities are discarded. As a result, one obtains the real-valued least-squares solution and corresponding variance matrices

$\left[\begin{array}{l}\hat{a} \\ \hat{b}\end{array}\right], \quad\left[\begin{array}{cc}Q_{\hat{a}} & Q_{\hat{a} \hat{b}} \\ Q_{\hat{b} \hat{a}} & Q_{\hat{b}}\end{array}\right]$

This solution is often referred to as the float solution.

In the second step, the results $\hat{a}$ and $Q_{\hat{a}}$ of the first step are used to compute the integer least-squares estimates of the ambiguities. The integer least-squares estimate of $a$ is denoted as $\check{a}$, and is the solution of

$\min _{a}(\hat{a}-a)^{T} Q_{\hat{a}}^{-1}(\hat{a}-a), \quad a \in Z^{n}$

where $Z^{n}$ is the $n$-dimensional space of integers. In two dimensions, we have $n=2$. Once the minimizer $\check{a}$ has been found, the residual $(\hat{a}-\check{a})$ is used to adjust the float solution $\hat{b}$. This is done in the third step. As a result the fixed solution $\breve{b}$ and its variance matrix are obtained as

$\check{b}=\hat{b}-Q_{\hat{b} \hat{a}} Q_{\hat{a}}^{-1}(\hat{a}-\check{a}), \quad Q_{\check{b}}=Q_{\hat{b}}-Q_{\hat{b} \hat{a}} Q_{\hat{a}}^{-1} Q_{\hat{a} \hat{b}}$

The computations needed for the first and third step are rather straightforward and can be based on standard techniques. Not so however for the second step. Due to the integer constraints on the ambiguities and the fact that the ambiguity variance matrix is non-diagonal, the solution of Eq. (3) must be obtained by means of a search. The idea is to replace the global search space of integers $Z^{n}$ by a local one, the so-called ambiguity search space. It reads

$(\hat{a}-a)^{T} Q_{\hat{a}}^{-1}(\hat{a}-a) \leq \chi^{2}$

It is centred at $\hat{a}$, its shape and orientation are governed by $Q_{\hat{a}}$ and its size can be controlled by $\chi^{2}$. The size is assumed to be set such that the sought integer leastsquares solution is indeed contained in the search space. The solution is then obtained by searching through the search space. The efficiency of the search is poor, however, when the search space is highly elongated, having principal axes that fail to coincide with the grid axes. The idea is therefore to replace the original integer least-squares problem of Eq. (3) by an equivalent one, but one that can be solved more efficiently. This is done in two steps. After replacing the original DD ambiguities by new ones, a search based on a sequential conditional least-squares adjustment is carried out. Both steps are based on the idea of decorrelation.

\subsection{Ambiguity decorrelation}

The original least-squares problem of Eq. (3) is reparametrized and replaced by the equivalent problem

$\min _{z}(\hat{z}-z)^{T} Q_{\hat{z}}^{-1}(\hat{z}-a), \quad z \in Z^{n}$

with

$\hat{z}=Z^{T} \hat{a}$ and $Q_{\hat{z}}=Z^{T} Q_{\hat{a}} Z$

In order for this transformed problem to be equivalent to the original problem, the matrix $Z$ needs to be integer and volume preserving (Teunissen 1995b). In two dimensions, preserving the volume means preserving the area of the search space. Once the integer leastsquares solution $\check{z}$ of the transformed problem has been found, the solution of the original problem is found through the back transformation $\check{a}=Z^{-T} \check{z}$.

Matrix $Z^{T}$ should be constructed in such a way that it decorrelates. That is, it should make $Q_{\hat{z}}$ more diagonal than the original ambiguity variance matrix $Q_{\hat{a}}$. Let the original two-dimensional ambiguity variance matrix be given as

$Q_{\hat{a}}=\left[\begin{array}{cc}\sigma_{a_{1}}^{2} & \sigma_{a_{1} a_{2}} \\ \sigma_{a_{2} a_{1}} & \sigma_{a_{2}}^{2}\end{array}\right]$

Let us first consider the case of a full decorrelation. A complete decorrelation is achieved by means of either one of the following two matrices

$T_{1}^{T}=\left[\begin{array}{cc}1 & -\sigma_{a_{1} a_{2}} \sigma_{a_{2}}^{-2} \\ 0 & 1\end{array}\right], \quad T_{2}^{T}=\left[\begin{array}{cc}1 & 0 \\ -\sigma_{a_{2} a_{1}} \sigma_{a_{1}}^{-2} & 1\end{array}\right]$

The resulting variance matrices are diagonal and read

$T_{1}^{T} Q_{\hat{a}} T_{1}=\left[\begin{array}{cc}\sigma_{a_{1} \mid a_{2}}^{2} & 0 \\ 0 & \sigma_{a_{2}}^{2}\end{array}\right], \quad T_{2}^{T} Q_{\hat{a}} T_{2}=\left[\begin{array}{cc}\sigma_{a_{1}}^{2} & 0 \\ 0 & \sigma_{a_{2} \mid a_{1}}^{2}\end{array}\right]$

where $\sigma_{a_{1} \mid a_{2}}^{2}$ and $\sigma_{a_{2} \mid a_{1}}^{2}$ are conditional variances. Note that the two matrices of Eq. (8) satisfy two out of the three necessary conditions. They both decorrelate and they are both area preserving. Their entries, however, are not all integer. In order to repair this situation, the idea is to replace them by their integer approximation. That is, both $\sigma_{a_{1} a_{2}} \sigma_{a_{2}}^{-2}$ and $\sigma_{a_{2} a_{1}} \sigma_{a_{1}}^{-2}$ are rounded to their nearest integer. This results in two matrices, which are still area preserving and now also integer. The full decorrelation property is lost however. In fact, a full 
decorrelation will very seldom be attainable by means of integer matrices. Still, we can reach a significant decorrelation if we use both matrices in a sequence, one after the other. This implies that we first replace the first ambiguity $a_{1}$ by a new ambiguity $a_{1}^{\prime}$, which is an integer linear combination of $a_{1}$ and $a_{2}$. Then we replace the second ambiguity $a_{2}$ by a new ambiguity $a_{2}^{\prime}$, which is an integer linear combination of $a_{1}^{\prime}$ and $a_{2}$. In each step, a decorrelation will occur. This process is then repeated until no further decorrelation is possible. Hence, matrix $Z^{T}$ is constructed from a sequence of transformations of the following two types

$Z_{1}^{T}=\left[\begin{array}{cc}1 & -z_{12} \\ 0 & 1\end{array}\right], Z_{2}^{T}=\left[\begin{array}{cc}1 & 0 \\ -z_{21} & 1\end{array}\right]$

in which $z_{12}$ and $z_{21}$ are in each step taken as the nearest integer to the appropriate ratio of ambiguity covariance and ambiguity variance. A geometric description of this decorrelating ambiguity transformation is given in Teunissen (1995a).

\subsection{Integer least-squares search}

Once the decorrelating ambiguity transformation has been constructed, the transformed search space can be formulated as

$(\hat{z}-z)^{T} Q_{\hat{z}}^{-1}(\hat{z}-z) \leq \chi^{2}$

By applying a conditional least-squares adjustment, we can write the quadratic form as a sum of squares

$\left(\frac{\hat{z}_{1}-z_{1}}{\sigma_{z_{1}}}\right)^{2}+\left(\frac{\hat{z}_{2 \mid 1}-z_{2}}{\sigma_{z_{2 \mid 1}}}\right)^{2} \leq \chi^{2}$

with the conditional least-squares estimate $\hat{z}_{2 \mid 1}=\hat{z}_{2}-\sigma_{z_{2} z_{1}} \sigma_{z_{1}}^{-2}\left(\hat{z}_{1}-z_{1}\right)$. The sum of squares allows us to describe the search space by means of bounds on the two individual ambiguities,

$\left\{\begin{array}{l}\left(\hat{z}_{1}-z_{1}\right)^{2} \leq \sigma_{z_{1}}^{2} \chi^{2} \\ \left(\hat{z}_{2 \mid 1}-z_{2}\right)^{2} \leq \sigma_{z_{2 \mid 1}}^{2} \chi^{\prime 2}\end{array}\right.$

with $\chi^{\prime 2}=\chi^{2}-\left(\hat{z}_{1}-z_{1}\right)^{2} / \sigma_{z_{1}}^{2}$. The search for the integer least-squares solution can now very briefly be described as follows. First one selects an integer ambiguity $z_{1}$ that satisfies the first bound. Then, based on this chosen integer value, the conditional least-squares estimate $\hat{z}_{2 \mid 1}$ and scalar $\chi^{\prime 2}$ are computed. These values are then used to select an integer ambiguity $z_{2}$ that satisfies the second bound. By repeating this process, admissible integer pairs $\left(z_{1}, z_{2}\right)$ are obtained, from which then the sought integer least-squares ambiguities can be chosen. More details on the intricacies of this conditional least-squaresbased search, including a geometric description and some useful variations, can be found in Teunissen (1993), Teunissen (1995a) and Jonge and Tiberius (1996).

The reasons why the preceding search is so much more efficient than when applied to the original DD ambiguities are twofold. First, search halting occurs repeatedly when the search would be executed in terms of the original DD ambiguities. This can be explained as follows. Since $\sigma_{a_{2} \mid a_{1}}^{2}=\sigma_{a_{2}}^{2}\left(1-\rho_{a_{1} a_{2}}^{2}\right)$, it follows, if the correlation coefficient $\rho_{a_{1} a_{2}}$ is close to one and the two ambiguity variances are about equal, that $\sigma_{a_{2} \mid a_{1}}^{2} \ll \sigma_{a_{1}}^{2}$. This implies, when the search is based on the use of the DD ambiguities, that the second bound of Eq. (13) is much sharper than the first bound, which in fact is rather loose due to the poor precision of the DD ambiguities. Thus the first bound admits quite some integer candidates, whereas the second bound does not. Hence, everytime an integer candidate is found for the first ambiguity $a_{1}$, a high likelihood exists of not being able to match it with an admissible integer for the second ambiguity $a_{2}$. This search halting is due to the poor precision of the DD ambiguities and the fact that they are highly correlated.

The second reason why the search is so much more efficient has to do with the chosen size of the search space. It will be clear that prior to the search, a value for the scale factor $\chi^{2}$ needs to be chosen. This value should not be too large, but also not too small. Too large a value implies that the search space would contain an abundance of unnecessary grid points. However, in order not to end up with an empty search space, the value should also not be too small. Due to the high precision and low correlation of the transformed ambiguities, it is possible to downsize the search space and still guarantee that it will contain at least one grid point, or if needed for validation, two grid points. In order to guarantee that the search space contains at least one single grid point, we proceed as follows. Starting from the realvalued least-squares estimate of the transformed ambiguities $\hat{z}$, we round each of its two entries to their nearest integer. This will give an integer vector, which then is substituted for $z$ into the quadratic form of Eq. (11). The value of $\chi^{2}$ is then taken to be equal to the value of the quadratic form. This approach guarantees that the search space will at least contain one grid point. Also the number of grid points contained in it will be small. This is due to the high precision and low correlation of the transformed ambiguities. In fact, it often happens that the search space so obtained only contains one grid point, since in many cases the rounded integer vector of $\hat{z}$ already equals the integer least-squares estimate $\check{z}$. This procedure takes full advantage of the transformed ambiguities. It will not work with the original DD ambiguities. Numerical examples showing how well the procedure works can be found in Teunissen et al. (1996).

\section{Does the widelane decorrelate?}

In the previous section we gave a brief outline of the steps involved in the least-squares ambiguity decorrelation adjustment; no mention has been made of the widelane. Witnessing the enormous literature on the topic of integer ambiguity estimation however, the widelane still plays a prominant role in many of the 
ambiguity fixing procedures that have been proposed and published. This is puzzling, in the sense that if the widelane has a role to play in the process of integer ambiguity estimation, it should fit into the theory of the previous section. In this section we will make a start by showing how the widelane relates to the concepts of the previous section.

\subsection{The widelane observable and the widelane ambiguity}

The observation equations on which our analysis will be based are those of the geometry-free model. For a single epoch $i$, they read as

$$
\begin{aligned}
& \phi_{1}(i)=\rho(i)-\mu_{1} I(i)+\lambda_{1} a_{1}+e_{\phi_{1}}(i) \\
& \phi_{2}(i)=\rho(i)-\mu_{2} I(i)+\lambda_{2} a_{2}+e_{\phi_{2}}(i) \\
& p_{1}(i)=\rho(i)+\mu_{1} I(i)+e_{p_{1}}(i) \\
& p_{2}(i)=\rho(i)+\mu_{2} I(i)+e_{p_{2}}(i)
\end{aligned}
$$

with $\phi_{1}(i)$ and $\phi_{2}(i)$, the DD phase observables on $L_{1}$ and $L_{2}$ at epoch $i$, expressed in units of range, rather than in cycles; $p_{1}(i)$ and $p_{2}(i)$, the corresponding code observables; $\rho(i)$, the DD form of the unknown ranges from receivers to satellites; $\lambda_{1}$ and $\lambda_{2}$, the known wavelengths of the $L_{1}$ and $L_{2}$ frequency; $a_{1}$ and $a_{2}$, the two unknown integer carrier phase ambiguities; $I(i)$, the unknown DD ionospheric delay at epoch $i ; \mu_{1}$ and $\mu_{2}$, the known wavelength ratios $\mu_{1}=\frac{\lambda_{1}}{\lambda_{2}}, \mu_{2}=\frac{\lambda_{2}}{\lambda_{1}}$; and, $e_{\phi_{1}}(i), e_{\phi_{2}}(i), e_{p_{1}}(i)$ and $e_{p_{2}}(i)$, the a priori residuals that contain the measurement noises and remaining unmodelled effects.

This model is referred to as geometry-free because it dispenses with the receiver-satellite geometry. The observation equations are therefore linear from the outset and hence no further linearization is needed. Note that the tropospheric delays have not been modelled explicitly in Eq. (14). The reason for this is that, when present, they would automatically get lumped with the range parameters $\rho(i)$. This implies that all unknown parameters in the model, except the range parameters $\rho(i)$, can be estimated free from tropospheric biases. Also note, since the equations are in DD form, that the data are based on using two receivers, both tracking the same two satellites. The two receivers may be in motion or may be stationary.

In many GPS applications, a prominent role is played by certain linear combinations of the original phase and/ or code observables. Depending on the application at hand, derived observables are formed with certain properties, such as being invariant for $\rho(i)$ or being invariant for $I(i)$. In relation to ambiguity fixing, it is often the widelane combination that acts in a prominent role. Examples can be found in Hatch $(1982,1989)$, Wübbena (1989), Allison (1991), Euler and Landau (1992), Goad (1992), Cocard and Geiger (1992), Bock (1996).

When one refers to the widelane combination, it is first of all important to make clear whether one refers to the widelane observable or to the widelane ambiguity. The widelane observable is defined as $\phi_{w}(i)=\left(\frac{\phi_{2}(i)}{\lambda_{2}}-\frac{\phi_{1}(i)}{\lambda_{1}}\right) /\left(\frac{1}{\lambda_{2}}-\frac{1}{\lambda_{1}}\right)$

whereas the widelane ambiguity is defined as

$a_{w}=a_{2}-a_{1}$

The observation equation of the widelane observable has the same structure as the observation equations of the two original phase obervables given in Eq. (14). It reads

$\phi_{w}(i)=\rho(i)+I(i)+\lambda_{w} a_{w}+e_{\phi_{w}}(i)$

in which $\lambda_{w}$ is referred to as the widelane wavelength. It relates to $\lambda_{1}$ and $\lambda_{2}$ as

$\frac{1}{\lambda_{w}}=\frac{1}{\lambda_{2}}-\frac{1}{\lambda_{1}}$

The structure of the widelane observation equation, together with the precision of the widelane observable and the magnitude of the coefficients in the observation equation, are usually used as arguments for using the widelaning technique in ambiguity fixing. That is, for the purpose of ambiguity fixing, one considers the widelaning technique useful since it produces a phase observable having a relatively long wavelength, together with a still reasonably small ionospheric delay and noise that is not too greatly amplified. It is contended by the present author that this reasoning, although not entirely false, is at least incomplete and inaccurate.

First we would like to make clear that the explicit use of the widelane observable does not bring in anything extra. In fact, one can do without it. It will be clear that all available information is contained in the observation equations of Eq. (14). It will also be clear that no loss of information is experienced when a one-to-one transformation is applied to the observables. Thus, if the following one-to-one transformation on the observables,

$\left[\begin{array}{l}\phi_{w}(i) \\ \phi_{2}(i)\end{array}\right]=\left[\begin{array}{cc}-\frac{\lambda_{w}}{\lambda_{1}} & \frac{\lambda_{w}}{\lambda_{2}} \\ 0 & 1\end{array}\right]\left[\begin{array}{l}\phi_{1}(i) \\ \phi_{2}(i)\end{array}\right]$

is applied to Eq. (14), we obtain the transformed observation equations

$$
\begin{aligned}
\phi_{w}(i) & =\rho(i)+I(i)+\lambda_{w}\left(a_{2}-a_{1}\right)+e_{\phi_{w}}(i) \\
\phi_{2}(i) & =\rho(i)-\mu_{2} I(i)+\lambda_{2} a_{2}+e_{\phi_{2}}(i) \\
p_{1}(i) & =\rho(i)+\mu_{1} I(i)+e_{p_{1}}(i) \\
p_{2}(i) & =\rho(i)+\mu_{2} I(i)+e_{p_{2}}(i)
\end{aligned}
$$

in which we recognize the widelane observable $\phi_{w}(i)$. This set of equations has the same information content as Eq. (14). Thus if a proper least-squares adjustment is carried out on the basis of this set, one will get a solution which is identical to the solution one would get when using Eq. (14). The two solutions will differ only when not all equations of Eq. (20) are used, or when the correlation between $\phi_{w}(i)$ and $\phi_{2}(i)$ is not properly taken into account. But in that case the solution will be of less quality, since some of the available information is 
then left out. Since a proper least-squares adjustment of either Eq. (14) or Eq. (20) will give identical solutions, there is no real advantage gained by using Eq. (20). In fact, with Eq. (20) one has to make sure that proper care is taken of the correlation that has been introduced.

Note that Eq. (20) has still been parametrized in terms of the original ambiguities $a_{1}$ and $a_{2}$. Let us now introduce the one-to-one parameter transformation

$\left[\begin{array}{l}a_{1} \\ a_{2}\end{array}\right]=\left[\begin{array}{cc}-1 & 1 \\ 0 & 1\end{array}\right]\left[\begin{array}{l}a_{w} \\ a_{2}\end{array}\right]$

When substituted into Eq. (20), we get

$$
\begin{aligned}
\phi_{w}(i) & =\rho(i)+I(i)+\lambda_{w} a_{w}+e_{\phi_{w}}(i) \\
\phi_{2}(i) & =\rho(i)-\mu_{2} I(i)+\lambda_{2} a_{2}+e_{\phi_{2}}(i) \\
p_{1}(i) & =\rho(i)+\mu_{1} I(i)+e_{p_{1}}(i) \\
p_{2}(i) & =\rho(i)+\mu_{2} I(i)+e_{p_{2}}(i)
\end{aligned}
$$

in which we recognize the widelane observation equation. Since the parameter transformation is one-to-one, we are still dealing with all the information content available. But now of course, since we are solving for a different set of parameters, their individual estimates together with their precision will differ from those of the original parameters. In the present case, this only holds for $a_{1}$ and $a_{w}$, since all other parameters remained the same. It is in this context that one should understand the potential usefulness of the widelaning technique. That is, by replacing one of the original ambiguities with the widelane ambiguity, one introduces a new parameter which generally will have a least-squares solution that differs from the least-squares solution of the parameter it replaced. Hence, also its precision will differ in general. And only when its precision is better than the precision of the parameter it replaced will the widelane ambiguity be an asset for the ambiguity fixing process. Its better precision will then make it easier to solve for its corresponding integer least-squares estimate.

With the relevance of the parameter transformation in mind, it will be clear that also in this context the widelane observable is not needed explicitly. That is, instead of using the parameter transformation Eq. (21) to transform Eq. (20) into Eq. (22), one can use it equally well to transform the original observation equations of Eq. (14) into

$$
\begin{aligned}
& \phi_{1}(i)=\rho(i)-\mu_{1} I(i)+\lambda_{1}\left(a_{2}-a_{w}\right)+e_{\phi_{1}}(i) \\
& \phi_{2}(i)=\rho(i)-\mu_{2} I(i)+\lambda_{2} a_{2}+e_{\phi_{2}}(i) \\
& p_{1}(i)=\rho(i)+\mu_{1} I(i)+e_{p_{1}}(i) \\
& p_{2}(i)=\rho(i)+\mu_{2} I(i)+e_{p_{2}}(i)
\end{aligned}
$$

Both sets of observation equations, (22) and (23), contain the same information and are parametrized in terms of the same parameters. Hence, their least-squares solutions are also identical.

The fact that both Eqs. (22) and (23) give identical solutions also shows why one has to be careful using such arguments as "the widelaning technique is useful, since it produces a phase observable having a relatively long wavelength, together with a still reasonably small ionospheric delay and a noise that is not too greatly amplified'. The real test for assessing the usefulness of the widelane ambiguity, or for that matter any other ambiguity that might be introduced, lies in the ambiguity variance matrix. It is in this matrix where all the various aspects of the model come together, such as the a priori precision of the observables, the presence or absence of the ionospheric delays and the magnitude of the coefficients of the design matrix. Hence, in order really to understand the potential usefulness of the widelane ambiguities, we need to know the complete ambiguity variance matrix.

\subsection{The ambiguity variance matrix}

In order to use a model which is sufficiently flexible as far as the ionospheric delays are concerned, we will model them as random variables. The use of an a priori weighted ionosphere has been discussed in, e.g., Wild and Beutler (1991), Schaer (1994) and Bock (1996). The sample values of the ionospheric delays can be taken from an externally provided ionospheric model, see e.g. Georgiadou (1994), Wild (1994), Wanninger (1995). In some applications it even suffices to take zero as the sample value. The a priori uncertainty in the ionospheric delays will be modelled through its variance being given as $s_{I}^{2}$. The value of $s_{I}^{2}$ depends in a large part on the interstation distance between the two receivers. Since the ionosphere decorrelates as function of the interstation distance, $s_{I}^{2}$ is at its maximum for baselines where the ionosphere is fully decorrelated, and it gets smaller the shorter the baselines become. For sufficiently short baselines, it can be taken equal to zero. A proposal on how to describe $s_{I}^{2}$ as a function of the interstation distance can be found in Bock (1996).

Modelling the ionospheric delays as random variables allows us to consider three versions of the geometry-free model. The version in which the ionospheric delays are assumed absent or known $\left(s_{I}^{2}=0\right)$, the version in which the ionospheric delays are assumed present, but completely unknown $\left(s_{I}^{2}=\infty\right)$, and the version in which the ionospheric delays are assumed present and known with uncertainty $\left(0<s_{I}^{2}<\infty\right)$. The first version will be referred to as the ionosphere-fixed model, the second as the ionosphere-float model and the third as the ionosphere-weighted model.

The variance matrix of the $L_{1}$ and $L_{2}$ ambiguities follows once one solves the model given by Eq. (14), or for that matter Eq. (20), in a least-squares sense. To obtain the solution for the ionosphere-weighted case, one of course has to include the ionospheric observation equations with corresponding variance $s_{I}^{2}$. Setting $s_{I}^{2}=0$ would then give the ionosphere-fixed solution and setting $s_{I}^{2}=\infty$ gives the ionosphere-float solution. Upon solving the geometry-free model in a least-squares sense, using $k$ epochs of data, the ambiguity variance matrix follows as

$Q_{\hat{a}}\left(s_{I}^{2}\right)=\alpha Q_{\hat{a}}(0)+(1-\alpha) Q_{\hat{a}}(\infty)$ 
with

$$
\left\{\begin{aligned}
Q_{\hat{a}}(0)= & \frac{1}{k} \Lambda^{-1}\left[\sigma_{\phi}^{2} I_{2}+\frac{1}{2} \sigma_{p}^{2} e_{2} e_{2}^{T}\right] \Lambda^{-1} \\
Q_{\hat{a}}(\infty)= & \frac{1}{k} \Lambda^{-1}\left[\left(\sigma_{\phi}^{2}+\sigma_{p}^{2}\right) I_{2}+\frac{2 \sigma_{p}^{2}\left(\mu_{1}+\mu_{2}\right)}{\left(\mu_{2}-\mu_{1}\right)^{2}}\right. \\
& \left.\times\left(e_{2} \mu^{T}+\mu e_{2}^{T}\right)\right] \Lambda^{-1}
\end{aligned}\right.
$$

and where $\sigma_{\phi}^{2}$ denotes the phase variance, $\sigma_{p}^{2}$ the code variance and $\Lambda=\operatorname{diag}\left(\lambda_{1}, \lambda_{2}\right), \quad e_{2}=(1,1)^{T^{p}}, \mu=\left(\mu_{1}\right.$, $\left.\mu_{2}\right)^{T}$. Note that we have given the ambiguity variance matrix an argument in order to discriminate between the three versions $0<s_{I}^{2}<\infty, s_{I}^{2}=0$ and $s_{I}^{2}=\infty$. Also note that the ambiguity variance matrix of the ionosphereweighted version equals a scalar weighted mean of the ambiguity variance matrices $Q_{\hat{a}}(0)$ and $Q_{\hat{a}}(\infty)$. The weight $\alpha$ in Eq. (24) is given as

$\alpha=\left[1+\frac{1}{2}\left(\mu_{2}-\mu_{1}\right)^{2} \frac{s_{I}^{2}}{\sigma_{p}^{2}}\right]^{-1}$

It is driven by the ratio of the a priori ionospheric variance and the code variance. Note that $2 \sigma_{p}^{2} /$ $\left(\mu_{2}-\mu_{1}\right)^{2}$ is the variance with which the ionosphere can be estimated from a single epoch of code data only.

In the previous section we already indicated that the efficiency of computing the integer least-squares solution of the DD ambiguities is hindered by the fact that they are highly correlated. Having their ambiguity variance matrix available, we are now in a position to show this. In case of the ionosphere-fixed version, the correlation coefficient of the $L_{1}$ and $L_{2}$ ambiguities follows from Eq. (25) as

$\rho_{a_{1} a_{2}}(0)=\frac{1}{\sqrt{1+2 \sigma_{\phi}^{2} / \sigma_{p}^{2}}}$

This shows, since the precision of the phase observables is so much better than that of the code observables, that the correlation coefficient must be very close to 1 . For a phase-code variance ratio of $10^{-4}$, we have $\rho_{a_{1} a_{2}}(0) \approx 0.9998$, which is very close to 1 indeed.

When we consider the ionosphere-float case, the correlation coefficient follows from Eq. (25) as for the whole range of ionospheric spatial decorrelation one can think of.

\subsection{The precision of the widelane ambiguity}

Now that we have the formulae for the three types of ambiguity variance matrix available, we are in a position to analyse whether the widelane ambiguity decorrelates or not. In order to do so, we first will establish that decorrelation, when it occurs, goes hand in hand with an improvement in precision.

Let the two original DD ambiguities $\left(a_{1}, a_{2}\right)$ be replaced through an area-preserving transformation by $\left(a_{1}^{\prime}, a_{2}\right)$. Thus the second ambiguity remains unchanged and the first is replaced by an integer linear combination of the two original ambiguities. Since the transformation is area preserving, the determinant of the original ambiguity variance matrix is identical to the determinant of the transformed ambiguity variance matrix. Hence, $\sigma_{a_{1}}^{2} \sigma_{a_{2}}^{2}-\sigma_{a_{1} a_{2}}^{2}=\sigma_{a_{1}^{\prime}}^{2} \sigma_{a_{2}}^{2}-\sigma_{a_{1}^{\prime} a_{2}}^{2}$. From this it follows that $\frac{\sigma_{a_{1}^{\prime}}^{2}}{\sigma_{a_{1}}^{2}}=\frac{1-\rho_{a_{1} a_{2}}^{2}}{1-\rho_{a_{1}^{\prime} a_{2}}^{2}} \Rightarrow\left\{\sigma_{a_{1}^{\prime}}^{2}<\sigma_{a_{1}}^{2} \Leftrightarrow \rho_{a_{1}^{\prime} a_{2}}^{2}<\rho_{a_{1} a_{2}}^{2}\right\}$

This shows that the ambiguity $a_{1}^{\prime}$ decorrelates if and only if its precision is better than the precision of the ambiguity it replaced. Interpreting the ambiguity $a_{1}^{\prime}$ as the widelane ambiguity, it follows that the question of decorrelation can be answered by concentrating on the precision of the widelane ambiguity. Since $\sigma_{a_{2}}^{2} \leq \sigma_{a_{1}}^{2}$ for both the ionosphere-fixed case and the ionosphere-float case, it follows from the weighted mean Eq. (24) that the inequality also holds true for the ionosphere-weighted case. Hence, in order to show whether or not the widelane ambiguity decorrelates, it suffices to show whether or not the widelane ambiguity is of a better precision than the $L_{2}$ ambiguity.

3.3.1 Ionosphere-weighted case $\left(0<s_{I}^{2}<\infty\right)$. From the weighted mean of ambiguity variance matrices Eq. (24), it follows that

$\rho_{a_{1} a_{2}}(\infty)=\left(\mu_{1}+\mu_{2}\right) / \sqrt{\left[\left(1+\frac{\sigma_{\phi}^{2}}{\sigma_{p}^{2}}\right) \frac{\left(\mu_{2}-\mu_{1}\right)^{2}}{2\left(\mu_{1}+\mu_{2}\right)}+2 \mu_{1}\right]\left[\left(1+\frac{\sigma_{\phi}^{2}}{\sigma_{p}^{2}}\right) \frac{\left(\mu_{2}-\mu_{1}\right)^{2}}{2\left(\mu_{1}+\mu_{2}\right)}+2 \mu_{2}\right]}$

This correlation coefficient is also very close to 1 . For a phase-code variance ratio of $10^{-4}$, we have $\rho_{a_{1} a_{2}}(\infty) \approx 0.9995$.

From the scalar weighted mean of Eq. (24), it follows that the correlation coefficient of the ionosphereweighted case interpolates between $\rho_{a_{1} a_{2}}(0)$ and $\rho_{a_{1} a_{2}}(\infty)$. The conclusion is reached, therefore, that the $L_{1}$ and $L_{2}$ ambiguities are indeed extremely correlated

$$
\begin{aligned}
\sigma_{a_{w}}^{2}\left(s_{I}^{2}\right) \leq \sigma_{a_{2}}^{2}\left(s_{I}^{2}\right) \Leftrightarrow \quad & \alpha\left[\sigma_{a_{w}}^{2}(0)-\sigma_{a_{2}}^{2}(0)\right] \\
& +(1-\alpha)\left[\sigma_{a_{w}}^{2}(\infty)-\sigma_{a_{2}}^{2}(\infty)\right] \leq 0
\end{aligned}
$$

If we can show that the terms within the square brackets are smaller or at the most equal to zero, it follows, since the weight $\alpha$ satisfies the bounds $0 \leq \alpha \leq 1$, that the 
precision of the widelane ambiguity is better than the precision of the two original ambiguities. It follows then that the widelane indeed decorrelates.

3.3.2 Ionosphere-fixed case $\left(s_{I}^{2}=0\right)$. For the case where the ionospheric delays are assumed absent or known, the variances of the $L_{2}$ ambiguity and the widelane ambiguity follow from Eq. (25) as

$\left\{\begin{array}{l}\sigma_{a_{2}}^{2}(0)=\frac{1}{k \lambda_{2}^{2}}\left[\sigma_{\phi}^{2}+\frac{1}{2} \sigma_{p}^{2}\right] \\ \sigma_{a_{w}}^{2}(0)=\frac{1}{k \lambda_{w}^{2}}\left[\sigma_{\phi}^{2}\left(\frac{\lambda_{w}^{2}}{\lambda_{1}^{2}}+\frac{\lambda_{w}^{2}}{\lambda_{2}^{2}}\right)+\frac{1}{2} \sigma_{p}^{2}\right]\end{array}\right.$

Note that the coefficient of the phase variance is largest for the widelane ambiguity, but that the coefficient of the code variance is largest for the $L_{2}$ ambiguity. It follows from Eq. (31) that

$\sigma_{a_{w}}^{2}(0) \leq \sigma_{a_{2}}^{2}(0) \quad \Leftrightarrow \quad \frac{\sigma_{\phi}^{2}}{\sigma_{p}^{2}} \leq\left(\mu_{1}-\frac{1}{2}\right) \approx 0.28$

This shows that it is the variance ratio of phase and code which is instrumental in deciding whether or not the widelane ambiguity has a better precision. The widelane ambiguity will have a precision that is poorer than that of the $L_{2}$ ambiguity, when the code data are sufficiently precise in relation to the precision of the phase data. But this will clearly not happen in practice. The phase data are so much more precise than the code data, that the above bound on the phase-code variance ratio is easily fulfilled.

3.3.3 Ionosphere-float case $\left(s_{I}^{2}=\infty\right)$. For the case where the ionospheric delays are assumed present and completely unknown, the variances of the $L_{2}$ ambiguity and the widelane ambiguity follow from Eq. (25) as

$$
\left\{\begin{array}{l}
\sigma_{a_{2}}^{2}(\infty)=\frac{1}{k \lambda_{2}^{2}}\left[\left(\sigma_{\phi}^{2}+\sigma_{p}^{2}\right)+\frac{4 \mu_{2}\left(\mu_{1}+\mu_{2}\right)}{\left(\mu_{2}-\mu_{1}\right)^{2}} \sigma_{p}^{2}\right] \\
\sigma_{a_{w}}^{2}(\infty)=\frac{1}{k \lambda_{w}^{2}}\left[\left(\sigma_{\phi}^{2}+\sigma_{p}^{2}\right)\left(\frac{\lambda_{w}^{2}}{\lambda_{1}^{2}}+\frac{\lambda_{w}^{2}}{\lambda_{2}^{2}}\right)-\frac{4\left(\mu_{1}+\mu_{2}\right)}{\left(\mu_{2}-\mu_{1}\right)^{2}} \sigma_{p}^{2}\right]
\end{array}\right.
$$

Again we note that the coefficient of the phase variance is largest for the widelane ambiguity, but that the coefficient of the code variance is largest for the $L_{2}$ ambiguity. It follows from Eq. (33) that

$$
\begin{aligned}
\sigma_{a_{w}}^{2}(\infty) & \leq \sigma_{a_{2}}^{2}(\infty) \Leftrightarrow \\
\frac{\sigma_{\phi}^{2}}{\sigma_{p}^{2}} & \leq\left(4 \mu_{1} \frac{\left(\mu_{1}+\mu_{2}\right)\left(\mu_{1}+\mu_{2}-1\right)}{\left(\mu_{2}-\mu_{1}\right)^{2}}-1\right) \approx 25.9
\end{aligned}
$$

This is a very loose bound on the phase-code variance ratio. It is much looser than our previous bound of Eq. (32) and it will only fail to hold when the precision of the code data is far better than the precision of the phase data. This, however, will never be the case. Thus also in the case of an ionosphere-float solution, will the widelane ambiguity have a precision that is better than the precision of the two original DD ambiguities. When combining Eqs. (32) and (34) with Eq. (30) we thus reach the conclusion that, for all practical purposes, the widelane ambiguity indeed decorrelates.

\section{How does the widelane fit in?}

Now that we know that the widelane indeed decorrelates, it is natural to ask the question how the widelane fits in with the theory as discussed in Sect. 2; that is, does the widelane ambiguity have a place in the decorrelating ambiguity transformation $Z^{T}$ ? And if it does, in what way does it fit in? In this section, we will provide answers to these two questions.

\subsection{The widelane as initialization?}

When working with the widelane, we have two options. Either the widelane ambiguity replaces the $L_{1}$ ambiguity, or it replaces the $L_{2}$ ambiguity. The two relevant transformations are, respectively,

$$
\left[\begin{array}{cc}
1 & -1 \\
0 & 1
\end{array}\right] \text { and }\left[\begin{array}{cc}
1 & 0 \\
-1 & 1
\end{array}\right]
$$

Actually, the first transformation replaces $a_{1}$ with $-a_{w}$. This change of sign is, however, not relevant. Note that both these transformations are members of the two transformations of Eq. (10), the ones that are used in the LAMBDA method. Thus in principle it is possible that the widelane ambiguity is encountered while the ambiguity transformation $Z^{T}$ is constructed. In order to find out whether or not this is the case, we first need to decide which one of the two transformations of Eq. (35) will give the largest decorrelation. Since both transformations are area preserving, the determinant of the variance matrix of $\left(\hat{a}_{w}, \hat{a}_{2}\right)$ will be identical to the determinant of the variance matrix of $\left(\hat{a}_{1}, \hat{a}_{w}\right)$. Hence, analogous to Eq. (29), we have

$$
\frac{\sigma_{a_{2}}^{2}}{\sigma_{a_{1}}^{2}}=\frac{1-\rho_{a_{1} a_{w}}^{2}}{1-\rho_{a_{2} a_{w}}^{2}} \Rightarrow\left\{\sigma_{a_{2}}^{2}<\sigma_{a_{1}}^{2} \Leftrightarrow \rho_{a_{2} a_{w}}^{2}<\rho_{a_{1} a_{w}}^{2}\right\}
$$

This shows that, since the $L_{2}$ ambiguity is more precise than the $L_{1}$ ambiguity, the largest decorrelation is achieved when $a_{1}$ is replaced by $a_{w}$. Hence we can concentrate our attention on the transformation of the first type

$Z_{1}^{T}=\left[\begin{array}{cc}1 & -z_{12} \\ 0 & 1\end{array}\right]$

In order for the LAMBDA method to encounter the widelane ambiguity, we need to show whether or not the nearest integer of $\sigma_{a_{1} a_{2}} \sigma_{a_{2}}^{-2}$ equals 1 . That is, whether or not $z_{12}=\left[\sigma_{a_{1} a_{2}} \sigma_{a_{2}}^{-2}\right]=1$, in which [.] denotes rounding to the nearest integer. 
4.1.1 Ionosphere-weighted case $\left(0<s_{I}^{2}<\infty\right)$. The integer $z_{12}$ is chosen equal to 1 when $\sigma_{a_{1} a_{2}}\left(s_{I}^{2}\right) \sigma_{a_{2}}^{-2}\left(s_{I}^{2}\right) \in\left(\frac{1}{2}, \frac{3}{2}\right)$. From the scalar weighted mean of Eq. (24), it follows that
Together with Eqs. (40) and (38), this implies that the integer $z_{12}$ will always be set equal to 1, irrespective of the amount of spatial decorrelation of the ionosphere, that is, irrespective of the length of baseline used. We

$$
\begin{cases}\sigma_{a_{1} a_{2}}\left(s_{I}^{2}\right)>\frac{1}{2} \sigma_{a_{2}}^{2}\left(s_{I}^{2}\right) & \Leftrightarrow \alpha\left[\sigma_{a_{1} a_{2}}(0)-\frac{1}{2} \sigma_{a_{2}}^{2}(0)\right]+(1-\alpha)\left[\sigma_{a_{1} a_{2}}(\infty)-\frac{1}{2} \sigma_{a_{2}}^{2}(\infty)\right]>0 \\ \sigma_{a_{1} a_{2}}\left(s_{I}^{2}\right)<\frac{3}{2} \sigma_{a_{2}}^{2}\left(s_{I}^{2}\right) & \Leftrightarrow \alpha\left[\sigma_{a_{1} a_{2}}(0)-\frac{3}{2} \sigma_{a_{2}}^{2}(0)\right]+(1-\alpha)\left[\sigma_{a_{1} a_{2}}(\infty)-\frac{3}{2} \sigma_{a_{2}}^{2}(\infty)\right]<0\end{cases}
$$

This shows that if the conditions are satisfied for both the ionosphere- fixed case and the ionosphere-float case, then they are also satisfied for the intermediate ionosphere-weighted case.

4.1.2 Ionosphere-fixed case $\left(s_{I}^{2}=0\right)$. The ratio of the $L_{1} / L_{2}$ covariance with the variance of $L_{2}$, follows from Eq. (25) as

$\frac{\sigma_{a_{1} a_{2}}(0)}{\sigma_{a_{2}}^{2}(0)}=\mu_{2} /\left[1+2 \frac{\sigma_{\phi}^{2}}{\sigma_{p}^{2}}\right]$

From this, it follows that

$$
\begin{cases}\sigma_{a_{1} a_{2}}(0)>\frac{1}{2} \sigma_{a_{2}}^{2}(0) & \Leftrightarrow \frac{\sigma_{\phi}^{2}}{\sigma_{p}^{2}}<\mu_{2}-\frac{1}{2} \approx 0.78 \\ \sigma_{a_{1} a_{2}}(0)<\frac{3}{2} \sigma_{a_{2}}^{2}(0) & \Leftrightarrow \frac{\sigma_{\phi}^{2}}{\sigma_{p}^{2}}>\frac{1}{3} \mu_{2}-\frac{1}{2} \approx-0.07\end{cases}
$$

Again we obtain bounds on the phase-code variance ratio. The second bound is, of course, always satisfied. But the first bound is also satisfied for all practical purposes. Hence we may conclude that, at least for the ionosphere-fixed version, $z_{12}$ will always be set equal to 1 .

4.1.3 Ionosphere-float case $\left(s_{I}^{2}=\infty\right)$. In case the ionospheric delays are assumed present and completely unknown, the ratio of the $L_{1} / L_{2}$ covariance with the variance of $L_{2}$, follows from Eq. (25) as have thus reached the remarkable conclusion that the decorrelating ambiguity transformation of the LAMBDA method is always automatically initialized through the widelane transformation. This is a pleasing result, since it shows how the popular widelane ambiguity fits into the theory of Sect. 2.

\subsection{Can one do better than the widelane?}

Now that we know the decorrelating ambiguity transformation $Z^{T}$ automatically gets initialized by means of the widelane ambiguity, the question that remains to be answered is: Is the next transformation step in the sequence that builds up $Z^{T}$ equal to the identity matrix? If it is, then the widelane ambiguity would give the largest decorrelation possible. If not, then further improvements are still possible.

Before we try to answer this question let us first consider the precision of the widelane ambiguity for the two extreme cases, ionosphere-fixed and ionospherefloat. We have seen that the widelane decorrelates and that its precision is better than the precision of either the $L_{1}$ or the $L_{2}$ ambiguity. It also follows from Eqs. (31) and (33) that the precision of the widelane ambiguity differs only a little when we consider the two extreme cases of $s_{I}^{2}=0$ and $s_{I}^{2}=\infty$. For the ratio of their standard deviations, we have $\sigma_{a_{w}}(\infty) / \sigma_{a_{w}}(0) \approx 1.008$. Hence, their precision is very close indeed. However, the amount of decorrelation which is achieved by the

$\frac{\sigma_{a_{1} a_{2}}(\infty)}{\sigma_{a_{2}}^{2}(\infty)}=\left[2 \frac{\left(\mu_{1}+\mu_{2}\right)}{\left(\mu_{2}-\mu_{1}\right)^{2}}\left(1+\mu_{2}^{2}\right)\right] /\left[\frac{\sigma_{\phi}^{2}}{\sigma_{p}^{2}}+1+4 \mu_{2} \frac{\left(\mu_{1}+\mu_{2}\right)}{\left(\mu_{2}-\mu_{1}\right)^{2}}\right]$

From this, it follows that

$$
\begin{cases}\sigma_{a_{1} a_{2}}(\infty)>\frac{1}{2} \sigma_{a_{2}}^{2}(\infty) & \Leftrightarrow \frac{\sigma_{\phi}^{2}}{\sigma_{p}^{2}}<4 \frac{\left(\mu_{1}+\mu_{2}\right)}{\left(\mu_{2}-\mu_{1}\right)^{2}}\left(\mu_{2}^{2}-\mu_{2}+1\right)-1 \approx 43.27 \\ \sigma_{a_{1} a_{2}}(\infty)<\frac{3}{2} \sigma_{a_{2}}^{2}(\infty) & \Leftrightarrow \frac{\sigma_{\phi}^{2}}{\sigma_{p}^{2}}>\frac{4}{3} \frac{\left(\mu_{1}+\mu_{2}\right)}{\left(\mu_{2}-\mu_{1}\right)^{2}}\left(\mu_{2}^{2}-3 \mu_{2}+1\right)-1 \approx-14.02\end{cases}
$$

It is clear that both these bounds on the phase-code variance ratio are satisfied. Hence, also for the ionosphere-float version, the integer $z_{12}$ will be set equal to 1 . widelane transformation differs considerably between the two cases. For the ionosphere-fixed case, we have $\rho_{a_{2} a_{w}}(0) \approx 0.9959$, while we get $\rho_{a_{2} a_{w}}(\infty) \approx 0.2290$ for 
the ionosphere-float case. Hence, the widelane transformation only marginally decorrelates when the baselines are short, but it gives a significant decorrelation when the baselines are long. This difference in amount of decorrelation already indicates that we cannot expect the follow up of the widelane transformation to be the same for all baseline lengths. Thus although the first step in the sequence of $Z^{T}$ is the same for all values of $s_{I}^{2}$, the following steps, provided they exist, will in all likelihood differ.

Following the initialization through the widelane, the next transformation in the sequence that builds up $Z^{T}$ is of the form

$Z_{2}^{T}=\left[\begin{array}{cc}1 & 0 \\ -z_{21} & 1\end{array}\right]$ with $\quad z_{21}=\left[\sigma_{a_{2} a_{w}} \sigma_{a_{w}}^{-2}\right]$

It will be clear that no further improvement after the widelane transformation is possible when $z_{21}$ turns out to be equal to zero. This is the case when $\sigma_{a_{2} a_{w}}$ $\sigma_{a_{w}}^{-2} \in\left(-\frac{1}{2}, \frac{1}{2}\right)$. Let us now consider what happens with the two extreme versions of the geometry-free model.

4.2.1 Ionosphere-fixed case $\left(s_{I}^{2}=0\right)$. When we apply the widelane transformation to the ambiguity variance matrix $Q_{\hat{a}}(0)$, it follows from Eq. (25) that

$\frac{\sigma_{a_{2} a_{w}}(0)}{\sigma_{a_{w}}^{2}(0)}=\left[\frac{\sigma_{\phi}^{2}}{\sigma_{p}^{2}}+\frac{1}{2}\left(1-\mu_{2}\right)\right] /\left[\frac{\sigma_{\phi}^{2}}{\sigma_{p}^{2}}\left(1+\mu_{2}^{2}\right)+\frac{1}{2}\left(1-\mu_{2}\right)^{2}\right]$

From this, it follows that

$$
\left\{\begin{array}{l}
\sigma_{a_{2} a_{w}}(0)<\frac{1}{2} \sigma_{a_{w}}^{2}(0) \quad \Leftrightarrow \frac{\sigma_{\phi}^{2}}{\sigma_{p}^{2}}>-0.5 \\
\sigma_{a_{2} a_{w}}(0)>-\frac{1}{2} \sigma_{a_{w}}^{2}(0) \Leftrightarrow \frac{\sigma_{\phi}^{2}}{\sigma_{p}^{2}}>-\frac{1}{2} \frac{\left(\mu_{2}-1\right)\left(\mu_{2}-3\right)}{\mu_{2}^{2}+3} \approx 0.05
\end{array}\right.
$$

4.2.2 Ionosphere-float case $\left(s_{I}^{2}=\infty\right)$. When we apply the widelane transformation to the ambiguity variance matrix $Q_{\hat{a}}(\infty)$, it follows from Eq. (25) that
In both cases, Eqs. (45) and (47), we observe that the first bound on the phase-code variance ratio is trivially fulfilled, whereas the second bound will not be satisfied with the currently available precision of code and phase. The conclusion is therefore, for both of these cases and thus also for the ionosphere-weighted case, that the ambiguity transformation of Eq. (43) will not reduce to the identity transformation. Hence it is indeed possible to do a better job than the widelane transformation does and, moreover, it is the least-squares ambiguity decorrelation adjustment of Sect. 2 that automatically produces the required optimal decorrelating ambiguity transformation $Z^{T}$.

To conclude, we will now give some examples of the decorrelating ambiguity transformation $Z^{T}$. They vary for varying values of the variance ratio of phase and code.

Example 1. Let us start with an unrealistically high precision for the code observable: $\sigma_{p}=3 \mathrm{~cm}$. With $\sigma_{\phi}=0.3 \mathrm{~cm}$, this gives a phase-code variance ratio of $\sigma_{\phi}^{2} / \sigma_{p}^{2}=0.01$. As a result we have for the ionospherefixed case, $\sigma_{a_{2} a_{w}} \sigma_{a_{w}}^{-2} \approx-1.98$ and thus $z_{21}=-2$. Hence, combined with the widelane transformation, this gives

$$
Z^{T}=\left[\begin{array}{ll}
1 & 0 \\
2 & 1
\end{array}\right]\left[\begin{array}{cc}
-1 & 1 \\
0 & 1
\end{array}\right]=\left[\begin{array}{ll}
-1 & 1 \\
-2 & 3
\end{array}\right]
$$

It can be shown that this is indeed the optimal decorrelating ambiguity transformation. The correlation coefficient of the transformed ambiguities equals $\rho_{z_{1} z_{2}}=0.01$. As remarked earlier, the sign of an individual ambiguity is not relevant. Hence, instead of having $a_{1}$ replaced by $a_{w}$ in the first step, one could also have it replaced by $-a_{w}$. In that case, the off-diagonal entry of the second transformation would be -2 instead of 2 .

Example 2. If we now choose instead of $3 \mathrm{~cm}$, a code standard deviation of $\sigma_{p}=10 \mathrm{~cm}$, the phase-code variance ratio reduces to $\sigma_{\phi}^{2} / \sigma_{p}^{2}=0.0009$. As a result we now have, $\sigma_{a_{2} a_{w}} \sigma_{a_{w}}^{-2} \approx-3.31$, and thus $z_{21}=-3$. Again it can be shown that only two transformation steps are needed. The final decorrelating ambiguity transformation therefore reads

$\frac{\sigma_{a_{2} a_{w}}(\infty)}{\sigma_{a_{w}}^{2}(\infty)}=\left[\left(\frac{\sigma_{\phi}^{2}}{\sigma_{p}^{2}}+1\right)-2 \frac{\left(\mu_{1}+\mu_{2}\right)}{\left(\mu_{2}-\mu_{1}\right)^{2}}\left(1-\mu_{2}\right)^{2}\right] /\left[\left(\frac{\sigma_{\phi}^{2}}{\sigma_{p}^{2}}+1\right)\left(1+\mu_{2}^{2}\right)-4 \frac{\left(\mu_{1}+\mu_{2}\right)}{\left(\mu_{2}-\mu_{1}\right)^{2}}\left(1-\mu_{2}\right)^{2}\right]$

From this, it follows that

$$
\begin{cases}\sigma_{a_{2} a_{w}}(\infty)<\frac{1}{2} \sigma_{a_{w}}^{2}(\infty) & \Leftrightarrow \frac{\sigma_{\phi}^{2}}{\sigma_{p}^{2}}>-1 \\ \sigma_{a_{2} a_{w}}(\infty)>-\frac{1}{2} \sigma_{a_{w}}^{2}(\infty) & \Leftrightarrow \frac{\sigma_{\phi}^{2}}{\sigma_{p}^{2}}>8 \frac{\left(\mu_{1}+\mu_{2}\right)\left(1-\mu_{2}\right)^{2}}{\left(\mu_{2}-\mu_{1}\right)^{2}\left(\mu_{2}^{2}+3\right)}-1 \approx 0.12\end{cases}
$$


$Z^{T}=\left[\begin{array}{ll}1 & 0 \\ 3 & 1\end{array}\right]\left[\begin{array}{cc}-1 & 1 \\ 0 & 1\end{array}\right]=\left[\begin{array}{ll}-1 & 1 \\ -3 & 4\end{array}\right]$

The correlation coefficient of the transformed ambiguities equals $\rho_{z_{1} z_{2}}=-0.32$. Remembering that the widelane transformation only resulted in a marginal decorrelation, the second step thus gives a considerable improvement over the first step.

Example 3. If we consider a further reduction in the phase-code variance ratio, say to the level of $\sigma_{\phi}^{2} / \sigma_{p}^{2}=10^{-4}$, then not two, but three transformation steps are used

$Z^{T}=\left[\begin{array}{cc}-1 & 1 \\ 0 & 1\end{array}\right]\left[\begin{array}{ll}1 & 0 \\ 4 & 1\end{array}\right]\left[\begin{array}{cc}-1 & 1 \\ 0 & 1\end{array}\right]=\left[\begin{array}{ll}-3 & 4 \\ -4 & 5\end{array}\right]$

Note that the widelane transformation appears twice. This confirms the discussion of Sect. 3.1, namely that the test of which integer linear combinations of the DD ambiguites are to be taken should be based on the complete ambiguity variance matrix and not on isolated arguments as to the a priori precision of the widelane observable and the magnitude of the coefficients in its observation equation. Also note that although the widelane transformation appears twice, neither one of the two transformed ambiguities equals the widelane ambiguity.

If, instead of having the pair $\left(a_{w}, a_{2}\right)$ after the first step, one would have used the pair $\left(-a_{w}, a_{2}\right)$, then the sequence of three transformations would read

$$
Z^{T}=\left[\begin{array}{ll}
1 & 1 \\
0 & 1
\end{array}\right]\left[\begin{array}{cc}
1 & 0 \\
-4 & 1
\end{array}\right]\left[\begin{array}{cc}
1 & -1 \\
0 & 1
\end{array}\right]=\left[\begin{array}{ll}
-3 & 4 \\
-4 & 5
\end{array}\right]
$$

The correlation coefficient of the transformed ambiguities equals $\rho_{z_{1} z_{2}}=-0.42$.

Example 4. In the ionosphere-float case we have $\sigma_{a_{2} a_{w}} \sigma_{a_{w}}^{-2} \approx-7.39$, when the phase-code variance ratio equals $\sigma_{\phi}^{2} / \sigma_{p}^{2}=10^{-4}$, and $\sigma_{a_{2} a_{w}} \sigma_{a_{w}}^{-2} \approx-7.42$, when the phase-code variance ratio equals $\sigma_{\phi}^{2} / \sigma_{p}^{2}=0.25 \times 10^{-4}$. In both cases, we have $z_{21}=-7$. It can be shown that in both cases also two transformation steps suffice. Hence, we have

$Z^{T}=\left[\begin{array}{ll}1 & 0 \\ 7 & 1\end{array}\right]\left[\begin{array}{cc}-1 & 1 \\ 0 & 1\end{array}\right]=\left[\begin{array}{cc}-1 & 1 \\ 7 & 8\end{array}\right]$

Although the decorrelating ambiguity transformation is the same for both cases, the decorrelation achieved is, of course, not the same. For the first case we have $\rho_{z_{1} z_{2}} \approx 0.0123$ and for the second case we have $\rho_{z_{1} z_{2}} \approx 0.0135$. Both cases are a considerable improvement on the correlation coefficient of 0.2290 of the first step.

\section{Summary}

In this contribution we studied the widelaning technique in relation to the theory of least-squares ambiguity decorrelation adjustment. For the benefit of analysing the widelaning technique, we brought together the relevant concepts in Sect. 2. It was emphasized that the computation of the integer least-squares DD ambiguities is often hindered because of their poor precision and very high correlation. That is why in the LAMBDA method use is made of ambiguities other than the DD ambiguities. The ambiguities that are used are in a one-to-one relation with the original DD ambiguities, but have, due to the way they are constructed, a much better precision and a much smaller correlation coefficient.

When studying the widelaning technique, it is important to understand the principles on which it is based. We therefore emphasized that the real test for the potential usefulness of the widelane should be based on the ambiguity variance matrix, rather than on isolated and often too vague arguments relating to the precision of the widelane observable and the magnitude of the coefficients in the observation equations. Since the ambiguity variance matrix is the vehicle used for evaluating the set of ambiguities, we presented the variance matrix of the least-squares DD ambiguities using the geometryfree model. We distinguished between three versions, which together cover short-, medium- and long-baseline applications.

In order to analyse how the widelaning technique fits into the theory of Sect. 2, we posed the following three questions: (1) Does the widelane ambiguity decorrelate? (2) Does the widelane ambiguity show up in the ambiguity transformation $Z^{T}$ ? (3) Can we do better than the widelane? It was shown that all three questions could be answered in the affirmative and that the widelane ambiguity indeed produces a smaller correlation coefficient in general. This holds true for all baseline lengths. It was also shown that the exceptions to this rule are not likely to be met in practice because of the high precision of the phase observables in relation to that of the code observables.

Since the widelane ambiguity could be shown to decorrelate, and since ambiguity decorrelation is the basic concept that lies at the root of the material of Sect. 2, the logical next step was to investigate whether the widelane ambiguity shows up in the decorrelating ambiguity transformation of the LAMBDA method. It was shown for each of the three versions of the geometryfree model and for the whole range of values the phasecode variance ratio may take in practice, that the widelane transformation is always the first step in the sequence that builds up the decorrelating ambiguity transformation. Hence, the LAMBDA method always gets automatically initialized with the widelane.

Finally, we inquired whether the use of the widelane ambiguity is the best one can do. This turned out not to be the case. In other words, the decorrelating ambiguity transformation goes beyond the widelane in its effort to obtain ambiguities which are maximally decorrelated. In the ionosphere-fixed case, for instance, the widelane ambiguity only achieves a change in the correlation coefficient from about 0.9998 to 0.9959 , whereas the complete decorrelating ambiguity transformation typically achieves a correlation coefficient in the order of 0.3 
to 0.4 . The widelane transformation does have a better record in the ionosphere-float case, bringing the correlation coefficient down from 0.9995 to 0.2290 . But also here a further improvement is achieved when using the decorrelating ambiguity transformation, bringing the correlation coefficient further down to about 0.01 .

One result of our analysis is that the rather old, but still popular widelaning technique has been cast and explained in the context of our theory of Sect. 2. A second is that we have shown that this technique is not explicitly needed. The widelaning technique is as initialization embedded in the LAMBDA method. Moreover, this method is capable of automatically improving upon the results achieved with the widelane. That is, once the ambiguity variance matrix is given, the method will be able to construct the optimal decorrelating ambiguity transformation automatically.

The limitations of the widelaning technique come even more to the fore if one considers models other than the geometry-free model, such as the geometry-based model, in which the relative receiver-satellite geometry is explicitly taken into account (Teunissen 1997). First, the widelaning technique requires the presence of dual-frequency data. Secondly, the widelaning technique does not have the capability to take the impact of the receiver-satellite geometry on the ambiguity variance matrix into account. Both these limitations are absent when using the method of Sect. 2. Examples thereof can be found in e.g. Teunissen (1994, 1995a), Jonge and Tiberius (1996).

Acknowledgements. This work was finalized during the author's stay as guest professor at the Department of Geodesy and Geomatics Engineering of the University of New Brunswick (Fredericton, Canada), with Professor A. Kleusberg as his host.

\section{References}

Allison T (1991) Multi-observable processing techniques for precise relative positioning. ION GPS-91, pp 715-725

Bock Y (1996) Medium-distance GPS measurements. In: Kleusberg A, Teunissen PJG (eds) GPS for Geodesy. Lecture Notes in Earth Sciences Vol 60. Springer, Berlin Heidelberg New York, pp 337-377

Blewitt G (1989) Carrier-phase ambiguity resolution for the Global Positioning System applied to geodetic baselines up to $2000 \mathrm{~km}$. J Geophys Res 94: 10187-10203

Cocard C, Geiger A (1992) Systematic search for all possible widelanes. Proc 6th Geod Symp Satellite Positioning, Columbus, Ohio, 17-20 March 1992

Counselman CC, Gourevitch SA (1981) Miniature interferometer terminals for earth surveying: ambiguity and multipath with Global Positioning System. IEEE Trans Geosci Rem Sens GE19: $244-252$
Euler H-J, Landau H (1992) Fast GPS ambiguity resolution onthe-fly for real-time applications. Proc 6th Geod Symp Satellite Positioning. pp 650-729

Frei E (1991) Rapid differential positioning with the Global Positioning System. Geodetic and Geophysical Studies in Switzerland Vol 44

Georgiadou Y (1994) Modelling the ionosphere for an active control network of GPS stations. LGR Series 7, Delft Geodetic Computing Centre

Goad CC (1992) Robust techniques for determining GPS phase ambiguities. Proc 6th Geod Symp Satellite Positioning. pp 245254

Hatch R (1982) The synergism of GPS code and carrier phase measurements. Proc 3rd Int Geod Symp Satellite Positioning, Las Vegas, New Mexico, Vol 2. pp 1213-1231

Hatch R (1989) Ambiguity resolution in the fast lane. ION GPS89: $45-50$

Jonge PJ de, Tiberius CCJM (1996) The LAMBDA method for integer ambiguity estimation: implementation aspects. $L G R$ Series 12, Delft Geodetic Computing Centre

Mervart L, Beutler G, Rothacher M, Wild U (1994) Ambiguity resolution strategies using the results of the International GPS Geodynamics Service (IGS). Bul Geod, 68: 29-38

Remondi BW (1986) Performing cm-level surveys in seconds with GPS carrier phase: initial results. Global Positioning System, Vol III. Institute of Navigation, pp 194-208

Schaer S (1994) Stochastische Ionospharenmodellierung beim 'Rapid Static Positioning' mit GPS. Astronomisches Institut, Universität Berne

Teunissen PJG (1993) Least-squares estimation of the integer GPS ambiguities. LGR Series 6, Delft Geodetic Computing Centre

Teunissen PJG (1994) A new method for fast carrier phase ambiguity estimation. Proc IEEE PLANS94. pp 562-574

Teunissen PJG (1995a) The least-squares ambiguity decorrelation adjustment: a method for fast GPS integer ambiguity estimation. J Geod 70: 65-82

Teunissen PJG (1995b) The invertible GPS ambiguity transformations. Manuscr Geod 20: 489-497

Teunissen PJG (1997) A canonical theory for short GPS baselines, part II: the ambiguity precision and correlation. J Geod: in print.

Teunissen PJG, Tiberius CCJM (1994) Integer least-squares estimation of the GPS phase ambiguities. Proc KIS94. pp 221-231

Teunissen PJG, Jonge PJ de, Tiberius CCJM (1996) The volume of the GPS ambiguity search space and its relevance for integer ambiguity resolution. Proc ION GPS-96, Kansas City, Missouri, September 17-20. pp 889-898

Wanninger L (1995) Improved ambiguity resolution by regional differential modelling of the ionosphere. Proc ION GPS-95, September 12-15, 1995, Palm Springs. pp 55-62

Wild U (1994) Ionosphere and geodetic satellite systems: permanent GPS tracking data for modelling and monitoring. Geodatisch- geophysikalische Arbeiten in der Schweiz, Band 48

Wild U, Beutler G (1991) Deterministische und stochastische Ionospharenmodelle. Z Vermess Photogr Kulturtech 6: 298-302

Wübbena G (1989) The GPS adjustment software package GEONAP - concepts and models. Proc 5th Geod Symp Satellite Positioning. pp 452-461

Wübbena G (1991) Zur Modellierung von GPS Beobachtungen für die hochgenaue Positionsbestimmung. PhD-thesis, TN-Hannover 